\title{
ON THE ANALYSIS OF LONGITUDINAL STRESS IN GLACIERS
}

\author{
By R. M. MCMEEKING and R. E. JOHNSON
}

(Department of Theoretical and Applied Mechanics, University of Illinois at Urbana-Champaign, Urbana, Illinois 61801, U.S.A.)

\begin{abstract}
In the standard solution for the stresses in a glacier or ice sheet obeying Glen's law, the down-slope component of the weight is supported by the basal shear stress, and the longitudinal deviatoric stress is second order. However, it has been found necessary to account for the longitudinal stress gradient when relating surface to bed topography with empirical data. In addition, during rapid stretching of the glacier, perhaps during a surge, the longitudinal stress gradient becomes comparable to or larger than the shear stress, and the standard solution is not entirely valid. In this paper, we consider the analysis of the stresses and strain-rates in a glacier when the longitudinal deviatoric stresses are comparable to the basal shear stresses. In some circumstances the down-slope component of weight is not borne completely by basal shear stress to leading order and some of the weight is shifted to the longitudinal deviatoric stress gradient. This case has also been examined. The results are used to obtain expressions for basal shear stress in terms of glacier thickness, slope, surface strain-rate gradient, and ice properties.
\end{abstract}

RÉSUMÉ. De l'analyse des contraintes longitudinales dans les glaciers. Dans la solution standard pour les contraintee dans un glacier ou une calotte de glace obéissant à la loi de Glen, la composante du poids vers l'aval est équilibrée par la contrainte de cisaillement à la base et la composante longitudinale du déviateur des contraintes est du second ordre. Cependant il est nécessaire de prendre en compte le gradient longitudinal des contraintes pour relier la topographie de surface à celle du lit avec des données empiriques. De plus, lors de rapides extensions du glacier, comme pour un surge, le gradient de contrainte longitudinal devient comparable ou supérieur à la contrainte de cisaillement et la solution standard n'est plus totalement valable. Dans cet article, nous envisageons l'analyse des contraintes et des defformations dans un glacier lorsque les

\section{INTRODUCTION}

In the standard solution for the flow of a glacier down a valley, the stress state is one of pressure plus a pure shear relative to Cartesian axes aligned with the mean slope. Other stress components such as longitudinal deviatoric stress are considered to be negligible compared to those retained. A discussion of the standard solution can be found in Raymond (1980) and the unique book by Hutter ([ $\left.{ }^{\left.c_{1} 1983\right]}\right]$ ). However, in some circumstances the longitudinal deviatoric stress magnitude is comparable to or exceeds the magnitude of the pure shear stress estimated in the standard solution. This would occur when the speed of the ice sliding over the bedrock is high. In particular, it seems likely that during a surge, the longitudinal deviatoric stress becomes large as the glacier stretches. This point is discussed and the results of this paper are used in a separate publication on the mechanics of surging glaciers (in preparation by the present authors). In addition, it has been found that it is necessary to take into account the longitudinal stress when using experimental measurements to relate surface and bed topography (Robin, 1967). Collins (1968), Budd contraintes longitudinales déviatrices sont comparables aux contraintes de cisaillement à la base. Dans certains cas la composante du poids vers l'aval n'est pas équilibrée totalement par la contrainte de cisaillement basale au premier ordre et une partie du poids est responsable d'un gradient longitudinal de contrainte. $\mathrm{Ce}$ cas a aussi été examiné. Les résultats sont utilisés pour obtenir des expressions pour la contrainte de cisaillement à la base en fonction de l'épaisseur de glace, de la pente, du gradient de déformation en surface et des propriétés de la glace.

Zusammenfassung. Über die Analyse der Längsspannung in Gletschern. In der Standardlösung für die Spannungen in Gletschern oder Eisschilden, die dem Glen'schen Fliessgesetz folgen, wird die hangabwärts gerichtete Komponente des Gewichts durch die Scherspannung am Untergrund aufgenommen, wăhrend die Ablenkungskraft in der Längsrichtung von 2. Ordnung ist. Es erwies sich jedoch als notwendig, den Längsspannungsgradienten $\mathrm{zu}$ berücksichtigen, wenn die Oberflächengestalt mit dem Untergrund über empirische Daten in Beziehung gesetzt werden soll. Ausserdem wird bei schnellen Beanspruchungen des Gletschers, etwa wăhrend eines Ausbruchs, der Längsspannungsgradient vergleichbar mit oder sogar grösser als die Scherung; dadurch verliert die Standardlösung ihre volle Gültigkeit. In dieser Arbeit stellen wir die Analyse der Spannungs- und Verformungsraten in einem Gletscher für den Fall dar, dass die ablenkende Lăngsspannungen den Scherspannungen am Untergrund vergleichbar sind. Unter gewissen Umständen wird die hangabwärts gerichtete Gewichtskomponente nicht vőllig durch die Scherspannung am Untergrund aufgenommen; ein Teil des Gewichts geht vielmer in den Gradienten der ablenkienden Läangsspannung ein. Dieser Fall wurde ebenfalls untersucht. Die Ergebnisse werden zur Aufstellung von Ausdrücken für die Scherspannung am Untergrun in Abhăngigkeit von Dicke, Gefälle, Verformungsrate an der Oberflăche und Eiseigenschaften herangezogen.

(1970), and Nye (1969) have all considered this problem, and use the governing equations for equilibrium of stress, the constitutive law, and averages through the thickness of the glacier to estimate the effect of longitudinal stress on the basal shear stress. Their result for the basal shear stress $\hat{\tau}_{b}$ takes the form

$$
\hat{\tau}_{\mathrm{b}}=\rho g \hat{h} \sin \alpha+G-T
$$

where $G$ is a function of the surface velocity, ice thickness $\hat{h}$, and material properties, and $T$ is a function of the shear-stress distribution ( $\alpha$ is the bed slope and $\rho g$ is the weight of the ice per unit volume). As noted by Nye (1969), some of the derivation leading to this result is exact, while other parts are questionable. Whereas the previous work has made relatively ad hoc attempts at determining $G$ and $T$, the present study determines them systematically and clarifies when the assumptions of the previous work are appropriate.

Hutter (1981) has obtained second-order corrections to the standard glacier solution by using a regular perturbation scheme and has obtained corrections for the longitudinal stress and shear stress. This derivation is 
valid when the ice flux due to basal sliding is comparable to or negligible compared with the ice flux due to shear flow. Therefore, it includes, as a special case, the case of no slip at the base which was considered by Nye (1969). Hutter's analysis avoids considering a separate boundary layer at the free surface (Johnson and McMeeking, 1984) by using a generalization to Glen's flow law which predicts linearly viscous behavior at low stress levels. There may be cases, however, when such an approach cannot be used to relate surface measurements to bottom topography. The reason for this is that a boundary-layer analysis may be necessary even when linear rheology is used to model the response to low stresses. Depending on the stress level at which the transition from power-law to linear rheology takes place, it is possible for the linearly viscous region near the free surface to be very small and embedded within the boundary layer. With regard to the present paper, however, a more serious limitation of Hutter's analysis is that when the ice flux due to basal sliding dominates the ice flux due to shear flow, the longitudinal stress magnitude is larger than that considered by Hutter. Fowler (1982) has considered this case, but restricted his attention to situations in which the shear stress still dominates the other deviatoric stresses.

In this paper, we are particularly interested in the following two cases: a glacier in which the longitudinal deviatoric stress is comparable to the shear stress at the base of the glacier, and a glacier in which the longitudinal stress is larger than the other stress components. We find that the state of stress in a glacier is dependent on the rate of sliding of the ice over the bedrock. It is conventional to assume that the speed of sliding is a function of the basal shear stress. This function may be monotonic for sliding without cavity formation or of the form shown in Figure 1 when cavities are present (Lliboutry, 1968; Hutter, 1982[b]). We

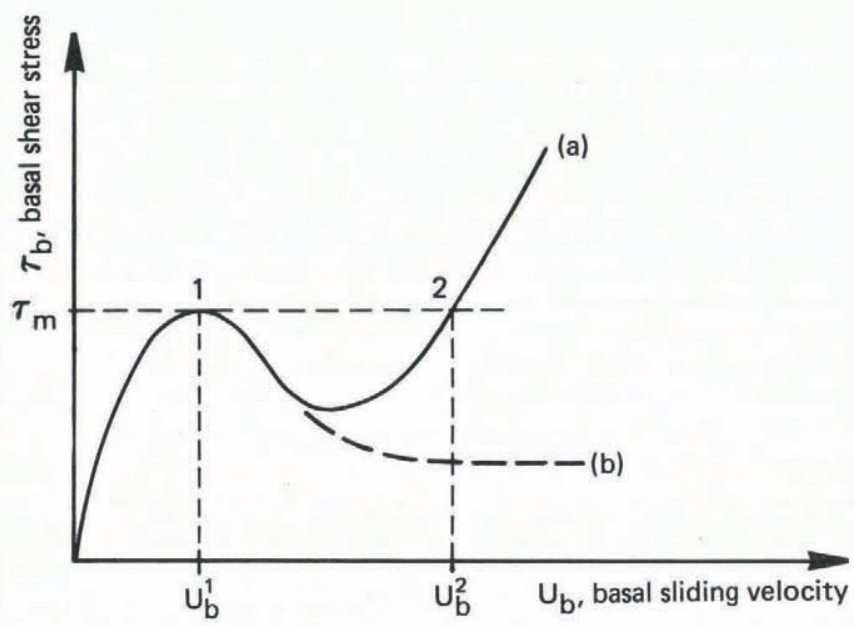

Fig. 1. Sliding law - relationship between basal shear stress and basal velocity.

find that the solution for portions of glaciers sliding at rates greater than $u_{b}^{1}$ (see Fig. 1) can be fundamentally different from portions sliding at lower speeds. At speeds lower than $u_{\mathrm{b}}^{1}$, the down-slope component of the weight can be supported by the basal shear stress to leading order. Above $u_{\mathrm{b}}^{1}$, some of the down-slope component of weight must be balanced by the longitudinal stress gradient to leading order and we find that the standard solution no longer serves as a basis for analysis.

In the next section, we present the governing equations for a glacier on a steep slope. In the following section, we consider the standard glacier solution which is valid for moderate basal sliding velocities. This is followed by two sections which discuss the analysis of glacier motion when the basal sliding velocity is large, i.e. when there is rapid stretching. This includes two cases in which the shear stress supports the glacier's weight and one case in which the longitudina deviatoric stress gradient supports part of the weight. Each case corresponds to a different magnitude of the basal sliding velocity and a different magnitude of the longitudinal deviatoric stress. We then obtain similar results for an ice mass resting on a gentle slope. Lastly, we consider the influence of the longitudinal stress on the basal shear stress for the cases studied in the previous sections.

\section{FORMULATION}

There have been numerous studies of the mechanics of glacial motion and a comprehensive review has been given by Hutter (1982[a], [ $\left.\left.{ }^{c} 1983\right]\right)$. Following Johnson and McMeeking (1984), we consider the gravity-driven flow of an incompressible viscous isotropic medium obeying Glen's law down a sloping valley with slowly varying bottom topography. The flow is two-dimensional (i.e. plane strain) and inertial effects are neglected. The slope of the free surface relative to the bottom is assumed to be small and the thickness of the glacier is small compared to its down-slope extent.

We introduce a Cartesian coordinate frame $(x, y)$ where the $x$-axis is along the mean bed line as in Figure 2. It should be noted that the coordinate system could also be aligned with the mean surface slope away

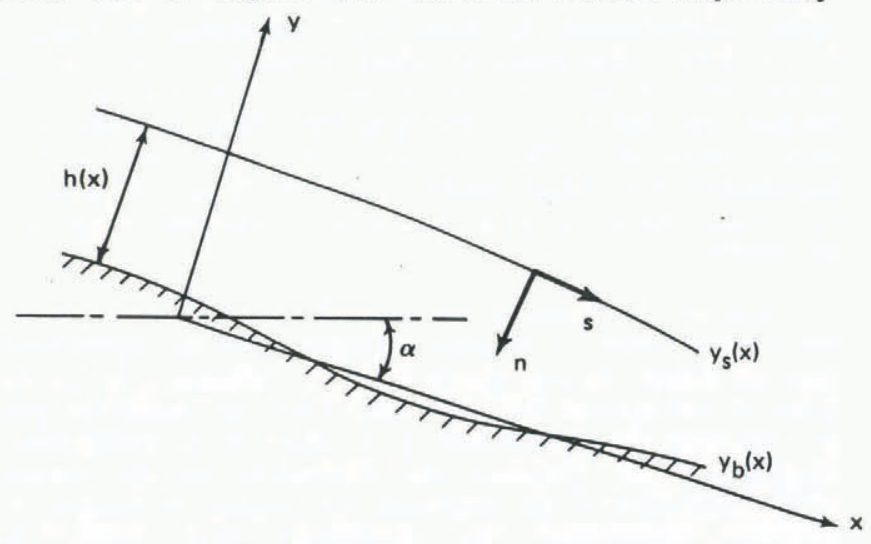

Fig. 2. A segment of the glacier showing the coordinate system.

from the margins, and the analysis would be unaltered. We first consider the case where the bed inclination angle $\alpha$ is assumed to be of order unity, i.e. steep glaciers, and introduce the non-dimensional variables $x=$ $x / L, y=\hat{y} / h_{0}, \mathbf{u}=\hat{\mathbf{u}} / \mathrm{U}, \hat{\boldsymbol{\sigma}} / \mathrm{\rho g} h_{0}$, where the circumflex indicates the physical variable. Here $h_{0}$ is the characteristic or maximum thickness of the glacier, $L$ is the length scale in the $\hat{x}$-direction which characterizes the length over which the steady-state flow field varies when driven only by time-independent accumulation and ablation representing averages over many years. In addition, $\mathbf{u}=(u, v)$ are the components of the velocity field, $\sigma_{i j}$ are the components of stress, $\rho$ is the uniform ice density, and $g$ is the gravitational constant. The deviatoric stresses $s_{i j}$ are introduced through the definitions $s_{i j}=\sigma_{i j}-1 / 2 \delta_{i j}\left(\sigma_{x x}+\sigma_{y y}\right)$, suitable for the incompressible plane flow of this problem.

Restricting attention to slow variations of the flow field due to steady (time averaged) accumulation and ablation, we require $\delta \equiv h_{0} / \mathrm{L} \ll 1$. In terms of the non-dimensional variables the governing equations become

$$
\begin{aligned}
\delta \frac{\partial \sigma_{x x}}{\partial x}+\frac{\partial \sigma_{x y}}{\partial y} & =-\sin \alpha \\
\delta & \frac{\partial \sigma_{x y}}{\partial x}+\frac{\partial \sigma_{y y}}{\partial y}=\cos \alpha,
\end{aligned}
$$




$$
\delta \frac{\partial u}{\partial x}+\frac{\partial v}{\partial y}=0 .
$$

Since the glacier is shallow or thin, we see from mass conservation, i.e. Equation (2), that the transverse velocity $v$ is small and of order $\delta$. The constitutive equation or flow law for the ice is taken to be Glen's law,

$$
\begin{aligned}
& \delta \frac{\partial u}{\partial x}=\tau^{n-1} s_{x x}, \frac{\partial v}{\partial y}=\tau^{n-1} s_{y y}, \\
& \frac{1}{2}\left[\frac{\partial u}{\partial y}+\delta \frac{\partial v}{\partial x}=\tau^{n-1} \sigma_{x y}\right.
\end{aligned}
$$

where $\tau^{2}=\frac{3}{2} \operatorname{tr}\left(\mathrm{s}^{2}\right)=3\left(s_{x x}^{2}+\sigma_{x y}^{2}\right)$ and $B$ is a material parameter which is often temperature dependent, but in this research we will study only isothermal conditions. The normalized form of Equation (3) results when the characteristic velocity $U$ is identified as $B h_{0}\left(\rho g h_{0}\right)^{n}$. We will carry out our analysis for $n=3$ which is characteristic of ice (Colbeck, 1980) and simplifies the analysis. The general features of the analysis for other values of $n$ (except $n=1$ ) will be the same.

The boundary conditions on the upper surface of the ice $y=y_{\mathrm{s}}(x)$ are those of zero traction. When trigonometric functions are expanded in terms of $y_{\mathrm{S}}^{\prime}(x)=$ $\mathrm{d} y_{\mathrm{s}} / \mathrm{d} x$, the conditions become (with reference to Figure 2)

$$
\begin{aligned}
\sigma_{n s} & =-\sigma_{x y}+2 \delta s_{x x} y_{s}^{\prime}(x)+ \\
& \left.+2 \delta^{2} \sigma_{x y}\left(y_{s}^{\prime}(x)\right)^{2}+o \delta^{3}\right)=0, \\
\sigma_{n n} & =\sigma_{y y}-2 \delta \sigma_{x y} y_{s}^{\prime}(x)+ \\
& \left.+2 \delta^{2} s_{x x}\left(y_{s}^{\prime}(x)\right)^{2}+O \delta^{3}\right)=0 .
\end{aligned}
$$

In addition, we have the kinematic boundary condition

$$
\frac{\partial h(x, t)}{\partial t}=Q(x, t)+v\left(\mathrm{x}, \mathrm{y}_{\mathrm{S}}\right)-\delta y_{\mathrm{S}}^{\prime}(x) u\left(x, y_{\mathrm{S}}\right)+O\left(\delta^{2}\right)
$$

where $Q$ is the normalized volume accumulation rate $\hat{Q} / U$ and $t=\hat{t} B\left(\rho g h_{0}\right)^{3}$.

At the base of the glacier $y=y_{\mathrm{b}}(x)$ we have zero normal velocity

$$
\begin{aligned}
v_{\mathrm{b}} & =v\left(x, y_{\mathrm{b}}\right)-\delta_{y_{\mathrm{b}}}(x) u\left(x, y_{\mathrm{b}}\right)- \\
& -\frac{1}{2} \delta^{2}\left(y_{\mathrm{b}}(x)\right)^{2} v\left(x, y_{\mathrm{b}}\right)+O\left(\delta^{3}\right)=0
\end{aligned}
$$

and a basal sliding law which we state in a general way as

$$
\begin{aligned}
u_{\mathrm{b}} & =u\left(x, y_{\mathrm{b}}\right)+\delta y_{\mathrm{b}}(x) v\left(x, y_{\mathrm{b}}\right)- \\
& -\frac{1}{2} \delta^{2}\left(y_{\mathrm{b}}(x)\right)^{2} u\left(x, y_{\mathrm{b}}\right)+O\left(\delta^{3}\right) \\
& =\Phi\left(\mathrm{s}\left(x, y_{\mathrm{b}}\right), x, t\right)
\end{aligned}
$$

where $\Phi$ is a prescribed function of $\mathrm{s}, x$, and $t$, and its dependence on $x$ and $t$ indicates that the bed can be inhomogeneous and conditions can vary with time, perhaps seasonally. The time dependence above is not intended to represent an explicit time dependence, but represents time dependence which would enter through the numerous physical mechanisms affecting $\Phi$, such as temperature, etc. We have assumed here that the sliding mechanism is basically dominated by viscous flow and hence $\Phi$ depends principally on the deviatoric stress. However, one generalization of this would be to include pressure dependence. For example, a sliding mechanism influenced by Coulomb friction would be likely to lead to this situation.

\section{STANDARD GLACIER SOLUTION}

The usual solution to Equations (1) to (8) will now be summarized. The details of the perturbation methods used and the results can be found in Hutter (1981) and Morland and Johnson (1980, 1982). Away from the upper surface

$$
\begin{aligned}
\sigma_{x y} & =\left(y_{\mathrm{S}}-y\right) \sin \alpha-\delta y_{\mathrm{S}}^{\prime}(x)\left(y_{\mathrm{s}}-y\right) \cos \alpha+\delta C_{1}(x)+O\left(\delta^{2}\right) \\
\sigma_{y y} & =\left(y-y_{\mathrm{s}}\right) \cos \alpha+\delta y_{\mathrm{s}}^{\prime}(x)\left(y_{\mathrm{s}}-y\right) \sin \alpha+C(\delta)^{2} \\
\sigma_{x x} & =\left(y-y_{\mathrm{s}}\right) \cos \alpha+2 \delta\left(\partial u^{(0)} / \partial x\right) /\left(3\left(y_{\mathrm{s}}-y\right)^{2} \sin ^{2} \alpha\right)+ \\
& +\delta y_{\mathrm{S}}^{\prime}(x)\left(y_{\mathrm{S}}-y\right) \sin \alpha+O\left(\delta^{2}\right)
\end{aligned}
$$

where $u^{(0)}$ is given in Equation (10) to follow. The function $C_{1}(x)$ is determined by matching with the boundary-layer solution valid near the free surface and is given by Johnson and McMeeking (1984) as

$$
C_{1}=\frac{\delta^{2 / 3} k \mathrm{~d}^{2} u_{\mathrm{S}}{ }^{(0)}(s, 0) / \mathrm{d} s^{2}}{\sin \alpha\left(\mathrm{d} u_{\mathrm{s}}{ }^{(0)}(s, 0) / \mathrm{d} s\right)^{1 / 3}}
$$

where the $(s, n)$ coordinate system is shown in Figure 2,

$$
k=\frac{1}{3 \sqrt{3}}\left[\frac{3}{2}\right]^{1 / 3} \mathrm{r}^{2}\left[\frac{1}{3}\right] / \mathrm{r}\left[\frac{2}{3}\right]
$$

and $\mathbf{r}$ is the gamma function. (Note that $C_{1}$ appearing in Johnson and McMeeking (1984) contains an error which we have corrected here.) The stretch rates in Equation (9a) are those tangential to the top surface at the surface. $C_{1}(x)$ represents a shear stress induced in the core of the glacier by the relatively large longitudinal stress $s_{x x}$ present in the near-surface region of the glacier.

The motion of the ice is such that

$$
\left.\begin{array}{rl}
u & =u^{(0)}(x, y)+O(\delta)=\Phi(h(x), x, t)+ \\
& +\frac{3}{2}\left[h^{4}-\left(y_{\mathrm{S}}-y\right)^{4}\right] \sin ^{3} \alpha+O(\delta),
\end{array}\right\}
$$

$$
v=-\delta \int_{y_{\mathrm{b}}}^{y} \frac{\partial u^{(0)}(x, y)}{\partial x} \mathrm{~d} y+\delta y_{\mathrm{b}}(x) u^{(0)}\left(x, y_{\mathrm{b}}\right)+O\left(\delta^{2}\right)
$$

In these equations we have recognized that the only non-zero deviatoric stress component at the base is $\sigma_{x y}$ at leading order and so to the same order the stress dependence of $\$$ will involve only $h$. That is so because $h$ determines the basal shear stress to leading order (see Equation (9)). Lastly, the solution is completed by the determination of the glacier's shape from the kinematic boundary condition (6).

The solution given by Equations (9) to (11) is consistent approximation only if the basal sliding law is such that $\Phi=O(1)$ or smaller. Furthermore, the variation of $\$$ with $x$ must be $O(1)$ or less as well. If these two conditions are met, then the stretch rate is consistent with $s_{x x}=O(8)$. In addition, an examination of the kinematic boundary condition reveals that it is desirable 
to choose $Q=O(\delta)$ for only then is it possible that steady states $(\partial h / \partial t=0)$ could exist consistent with this solution when $Q \neq 0$. In fact, the magnitude of $\delta$ can be set by defining $\delta q(x, t)=Q(x, t)$ where $q=O(1)$ and it would be sensible to choose $q$ to have a maximum value of 1 when averaged over several years. Thus

$$
\delta=\hat{\bar{Q}}_{\max } / B h_{\mathrm{o}}\left(\rho g h_{\mathrm{o}}\right)^{3}
$$

where $\hat{\bar{Q}}$ is the average of $\hat{Q}$ over several years. It follows that a steady state for the glacier shape averaged over several years can be determined from the kinematic condition by requiring that $\partial h / \partial t$ is $O\left(\delta^{2}\right)$ and therefore Equation (6) becomes

$$
\frac{\partial}{\partial x}\left[h\left(\Phi(h(x), x)+\frac{6}{5} h^{4} \sin ^{3} \alpha\right)\right]=\bar{q}(x)
$$

where $\bar{\Phi}$ is $\Phi$ averaged over several years not including a surge year. Additional in-depth discussion of the kinematic boundary condition and its role in determining the shape of the glacier can be found in Morland and Johnson (1980, 1982). Furthermore, note that the standard solution discussed in this section describes a flow whose spatial variation in the direction of the mean bed line is gentle. Consequently, this solution generally fails to be an accurate description of the flow near the margins of the glacier where the slope of the surface may become large. However, with some restrictions placed on $\Phi$, solutions valid at the margins can be found. Restrictions on the asymptotic behavior of $\Phi$ near the margins which lead to valid margin solutions have been investigated by Morland and Johnson (1980, 1982). The present analysis is primarily concerned with the central region of the glacier and therefore we simply assume that $\Phi$ has a form which permits a valid solution near the margin or that the small margin region does not significantly affect the solution valid away from the margin, i.e. the margin region is passive.

We note at this stage that the deviatoric stress $s_{x x}$ is much smaller than $\sigma_{x y}$, except near the upper surface. To see how the magnitude of $s_{x x}$ can be increased we must seek ways of increasing the stretch rate $\partial u / \partial x$. From Equation (10) we see that this can be done by increasing the magnitude of $\mathrm{d} \Phi / \mathrm{d} x$ to larger than $O(1)$ or by inducing rapid variations of $y_{\mathrm{s}}$ with $x$ perhaps by a sudden local accumulation of ice or snow. In the latter case, $s_{x x}$ would become comparable to $\sigma_{x y}$ only when the fluctuations of $y_{\mathrm{s}}$ were so rapid with $x$ that the perturbation scheme would break down. In any case, it is unlikely that such rapid local accumulations would take place in practice. Consequently, we confine our attention to the case where the rapid stretching is induced by the sliding law.

\section{RAPID STRETCHING: SHEAR STRESS SUPPORTS WEIGHT}

We will consider the bed on which the ice slides to be such that the dimensionless basal velocity is of order $1 / v$, i.e. $O(1 / v)$, where $v \ll 1$. For this to be so, the magnitude of the function $\Phi$ must be $O(1 / v)$ and we will rewrite it as $(1 / v)_{\Phi}$ where $\Phi=O(1)$. The magnitude of the basal velocity will determine the magnitude of the velocity in the ice as a whole and so we will consider an expansion of the form

$$
u(x, y)=\frac{1}{v} u^{(-1)}(x, y)+u^{(0)}(x, y)+O(1) .
$$

From this and the flow law Equation (3), it can be seen that

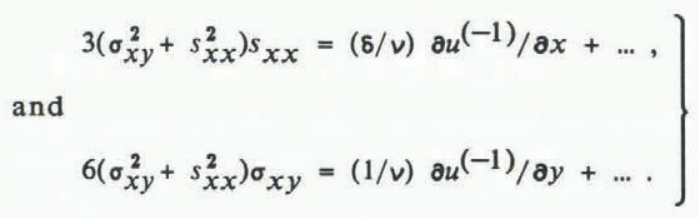

Furthermore, the equilibrium Equation (1a) implies that $\sigma_{x y}=O(1)$. Therefore, if $\delta / \nu$ is less than or equal to order unity, i.e. $\delta / v \leqslant O(1)$, then Equation (15) implies that $s_{x x}=O(\delta / v)$ and that $\partial u(-1) / \partial y \approx 0$. Hence $u^{(-1)}=$ $u^{(-1)}(x)$. Once this has been established, we see that for $\delta / v \leqslant O(1)$,

$$
\left.\begin{array}{l}
3\left(\sigma_{x y}^{2}+s_{x x}^{2}\right) s_{x x}=(\delta / v) \mathrm{d} u^{(-1)} / \mathrm{d} x \\
6\left(\sigma_{x y}^{2}+s_{x x}^{2}\right) \sigma_{x y}=\partial u^{(0)} / \partial y+\ldots,
\end{array}\right\}
$$

and the expansions for the stresses have the form

$$
\begin{aligned}
& \sigma_{x y}=\sigma_{x y}^{(0)}+\delta \sigma_{x y}^{(1)}+O(\delta), \\
& s_{x x}=(\delta / v) s_{x x}^{(0)}+\delta s_{x x}^{(1)}+O(\delta) .
\end{aligned}
$$

(a) Longitudinal deviatoric stress small compared to the shear stress

It now becomes necessary to consider the magnitude of $\delta / v$ versus 1 . In order to give a complete discussion of the solution regimes, first consider $\delta / v \ll 1$, i.e. $\delta \ll$ $v \ll 1$. This case has been considered by Fowler (1982) and from Equations (17) and (18) we see that the solution is still dominated by shear stress, since $\sigma_{x y} \gg$ $s_{x x}$. The solution is given by

$$
\begin{aligned}
& \sigma_{x y}^{(0)}=\left(y_{\mathrm{s}}-y\right) \sin \alpha, \\
& \sigma_{y y}=\sigma_{y y}^{(0)}+O(1)=\left(y-y_{\mathrm{s}}\right) \cos \alpha+O(1), \\
& s_{x x}^{(0)}=(\mathrm{d} \Phi / \mathrm{d} x) /\left[3\left(y_{\mathrm{s}}-y\right)^{2} \sin ^{2} \alpha\right]
\end{aligned}
$$

and of course $\sigma_{x x}=\sigma_{y y}+2 s_{x x}$. Because the deviatoric stress is $\sigma_{x y}$ only to leading order, the function $\Phi$ will depend on the shear stress at the glacier bed, the position, and time only. From Equations (16), (19), and boundary condition (8) the ice velocity is given by

$$
\begin{aligned}
u= & (1 / v) u^{(-1)}(x)+u^{(0)}(x, y)+O(1), \\
v= & (\delta / v)\left[y_{\mathrm{b}}(x) u^{(-1)}+\left(y_{\mathrm{b}}-y\right) \mathrm{d} u^{(-1)} / \mathrm{d} x\right]- \\
& -\delta \int_{y_{\mathrm{b}}}^{y} \frac{\partial u^{(0)}}{\partial x} \mathrm{~d} y+O(\delta)
\end{aligned}
$$

where

$$
u^{(-1)}=\Phi\left(\sigma_{x y}^{(0)}\left(x, y_{\mathrm{b}}\right), x, t\right)
$$

and

$$
u^{(0)}=\frac{3}{2}\left[h^{4}-\left(y_{s}-y\right)^{4}\right] \sin ^{3} \alpha .
$$

The kinematic condition leads to

$$
\begin{aligned}
& \frac{\partial h(x, t)}{\partial t}=-(\delta / v)\left[h \mathrm{~d} u^{(-1)} / \mathrm{d} x+\right. \\
& \left.+y_{\mathrm{S}}^{\prime}(x) u^{(-1)}\right]+\delta q(x, t)+O(\delta) .
\end{aligned}
$$

Because $\delta / v$ and $\delta$ are of different orders of magnitude, it follows that if $q=O(1)$, such a case generally cannot be in equilibrium with the climate and will thin and 
thicken relatively rapidly until the leading term in $\partial h / \partial t$ disappears. Such behavior has been studied by Fowler (1982), who analyzed the kinematic waves that the process would involve. If $q=O(1 / v)$, a steady-state solution with $\partial h / \partial t=O(\delta)$ could exist. As before, the complete solution might require a boundary layer near the margin. Note also from Equations (19) that there is a singularity in $s_{x \alpha}^{(0)}$ at $y=y_{S}$ and so the boundarytayer formulation of Johnson and McMeeking (1984) must be used near the surface.

(b) Longitudinal deviatoric stress comparable to the shear stress

The previous case $(\delta \ll v \ll 1)$ involves a situation in which the shear stresses $\sigma_{x y}$ are much larger than the longitudinal deviatoric stresses $s_{x x}$. Thus it would not seem to be suitable for describing the mechanics of surging, where in our work in preparation on surging glaciers we have estimated $s_{x x}$ to be comparable with $\sigma_{x y}\left(x, y_{\mathrm{b}}\right)$. This has been concluded also by Fowler (1982) for the different reason that the glacier would merely generate kinematic waves in response to seasonal changes. Furthermore, the expansion would break down and the solution would be inappropriate if $\mathrm{d} \Phi / \mathrm{d} x$ became very large anywhere and such a phenomenon is of ten suggested as the basis for surging models. Instead, we will consider the case in which $v=O(8)$ and, without losing any generality, we can identify $v$ with $\delta$. This corresponds to a situation in which the magnitude of the longitudinal deviatoric stress is comparable to that of the shear stress.

In this situation, the stresses would have the form

$$
\begin{gathered}
\sigma=\sigma^{(0)}+\delta \sigma^{(1)}+O\left(\delta^{2}\right) \\
\text { and } u=(1 / \delta) u^{(-1)}(x)+u^{(0)}(x, y)+O(\delta) \\
v=v^{(0)}(x, y)+\delta v^{(1)}(x, y)+O\left(\delta^{2}\right) .
\end{gathered}
$$

\section{As a consequence}

$$
\begin{aligned}
\tau^{2} & =3\left(\sigma_{x y}^{(0)^{2}}+s_{x x}^{(0)^{2}}\right)+6 \delta\left(\sigma_{x y}^{(0)} \sigma_{x y}^{(1)}+\right. \\
& \left.+s_{x x}^{(0)} s_{x x}^{(1)}\right)+O\left(\delta^{2}\right)
\end{aligned}
$$

and the flow law gives

$$
\left.\begin{array}{c}
\mathrm{d} u^{(-1)} / \mathrm{d} x=3 s_{x x}^{(0)^{3}}+3 \sigma_{x y}^{(0)^{2}} s_{x x}^{(0)}, \\
\partial u^{(0)} / \partial x=3\left(\sigma_{x y}^{(0)^{2}}+3 s_{x x}^{(0)^{2}}\right) s_{x x}^{(1)}+ \\
+6 \sigma_{x y}^{(0)} s_{x x}^{(0)} \sigma_{x y}^{(1)}, \\
\partial u^{(0)} / \partial y=6\left(\sigma_{x y}^{(0)^{3}}+s_{x x}^{(0)^{2}} \sigma_{x y}^{(0)}\right) .
\end{array}\right\}
$$

From the boundary conditions on $y=y_{\mathrm{S}}$ we find

$$
\begin{gathered}
\sigma_{x y}^{(0)}\left(x, y_{\mathrm{s}}\right)=0, \sigma_{x y}^{(1)}\left(x, y_{\mathrm{s}}\right)=2 y_{\mathrm{s}}^{\prime} s_{x x}^{(0)}\left(x, y_{\mathrm{s}}\right), \\
\sigma_{y y}^{(0)}\left(x, y_{\mathrm{s}}\right)=0, \quad \sigma_{y y}^{(-1)}\left(x, y_{\mathrm{s}}\right)=0,
\end{gathered}
$$

and on $y=y_{\mathrm{b}}$

$$
v^{(0)}\left(x, y_{\mathrm{b}}\right)=0, \quad v^{(1)}\left(x, y_{\mathrm{b}}\right)=y_{\mathrm{b}}(x) u^{(-1)}(x),
$$

$$
\begin{aligned}
& u^{(-1)}(x)=\Phi\left(\mathrm{s}^{(0)}, x, t\right), \\
& u^{(0)}\left(x, y_{\mathrm{b}}\right)=\frac{\partial \Phi\left(\mathrm{s}^{(0)}, x, t\right)}{\partial s_{i j}} s_{i j}^{(1)} .
\end{aligned}
$$

The equilibrium Equations (2) become at first and second order, respectively

$$
\frac{\partial \sigma_{x y}^{(0)}}{\partial y}=-\sin \alpha, \frac{\partial \sigma_{y y}}{\partial y}=\cos \alpha .
$$

and

$$
\frac{\partial \sigma_{X y}^{(1)}}{\partial y}=-\frac{\partial \sigma_{X X}^{(0)}}{\partial x}, \quad \frac{\partial \sigma_{y y}^{(1)}}{\partial y}=-\frac{\partial \sigma_{X y}^{(0)}}{\partial x} .
$$

We find by integrating Equations (31) using boundary conditions (27) and (28) that

$\sigma_{x y}^{(0)}=\left(y_{\mathrm{s}}-y\right) \sin \alpha$ and $\sigma_{y y}^{(0)}=\left(y-y_{\mathrm{s}}\right) \cos \alpha$

as the down-slope component of weight is still supported by the shear stress.

The boundary condition (30) and (25) together provide an equation for $s_{x x}^{(0)}$

$$
3 s_{x x}^{(0)^{3}}+3 \sigma_{x y}^{(0)^{2}} s_{x x}^{(0)}-\mathrm{d} u^{(-1)} / \mathrm{d} x=0 .
$$

Here we will use the conventional assumption that the sliding velocity at the base depends only on the basal shear stress. In this case $u^{(-1)}(x)$ will be known in terms of the bed shear stress and therefore in terms of the profile of the glacier. Consequently, $\mathrm{d} u^{(-1)} / \mathrm{d} x$ is then known and Equation (34) is a cubic algebraic equation for $s_{x x}^{(0)}$, which is easily solved. Note, however, that if we generalize the basal sliding law such that the base velocity also depends on the longitudinal deviatoric stress (which in the present analysis is of the same order as $\left.\sigma_{x y}\right)$, then $u^{(-1)}$ is a function of $s_{x x}\left(x, y_{\mathrm{b}}\right)$ and Equation (34), when evaluated at the bed $y=y_{\mathrm{b}}$, is a first-order, non-linear ordinary differential equation for the longitudinal deviatoric stress at the bed $s_{x x}^{(0)}\left(x, y_{\mathrm{b}}\right)$. This would be the case when $\Phi$ depends on $\tau$, the equivalent shear stress and the basal shear stress, rather than on the basal shear stress alone. For flow of ice over protrusions on the base, viscous flow at the base is likely to be an important sliding mechanism and therefore it does seem that $T$ would enter the sliding law. The value of $s_{x x}^{(0)}$ away from the base would be determined by solving Equation (34) as a cubic equation once the value of $s_{x x}^{(0)}\left(x, y_{\mathrm{b}}\right)$ and thus $\mathrm{d} u(-1) / \mathrm{d} x$ is known. Since the function $s_{x x}^{(0)}\left(x, y_{\mathrm{b}}\right)$ would be the solution of a differential equation, the basal sliding velocity $u^{(-1)}$ will depend on conditions everywhere along the base and not just on the local aspects. Budd (1975) has suggested that the local rate of sliding depends on conditions everywhere in the glacier, but has not presented any mechanical model as clear as ours. Furthermore, the consequences of this possibility have not been explored in the literature on the mechanics of glaciers, except in the heuristic treatment by Budd (1975). We will not attempt to expand on this topic, except to say that it seems possible that novel dynamic behavior could arise from this feature as Budd has found in his surging models. We will also not consider a pressure-dependent sliding law, except to note that such extensions are relatively straightforward.

Returning to the conventional case where Equation (34) is simply a cubic algebraic equation, we find it has only one real root

$$
s_{X X}^{(0)}=-\left[r+\sqrt{r^{2}+p^{3}}\right]^{1 / 3}-\left[r-\sqrt{r^{2}+p^{3}}\right]^{1 / 3}
$$

where $r=-\frac{1}{6} \mathrm{~d} u^{(-1)} / \mathrm{d} x$ and $p=\frac{1}{3} \sigma_{x y}^{(0)^{2}}$.

The second-order equilibrium Equation (32) can be integrated to give 


$$
\begin{gathered}
\sigma_{y y}^{(1)}=y_{\mathrm{S}}^{\prime}(x)\left(y_{\mathrm{S}}-y\right) \sin \alpha \\
\sigma_{x y}^{(1)}=y_{\mathrm{S}}^{\prime}(x)\left[\left(y-y_{\mathrm{S}}\right) \cos \alpha+\right. \\
\left.+4 s_{x x}^{(0)}\left(x, y_{\mathrm{S}}\right)-2 s_{x x}^{(0)}(x, y)\right]+ \\
+\frac{s_{x x}^{(0)}\left(x, y_{\mathrm{S}}\right)}{\frac{\partial\left|s_{x x}^{(0)}\left(x, y_{\mathrm{S}}\right)\right|}{\partial x}}\left[2^{1 / 6} z^{1 / 2}+6 z^{1 / 6} / \sqrt{2}+I_{1}\right]
\end{gathered}
$$

where $z=\sqrt{a\left(y_{\mathrm{S}}-y\right)^{6}+1}-1$,

$$
\begin{gathered}
a=\frac{4}{27}\left[\frac{\sin \alpha}{\mathrm{s}_{x x}^{(0)}\left(x, y_{s}\right)}\right]^{6}, \\
I_{1}(z)=\int_{0}^{z}\left\{\frac{1}{2^{5 / 6} x^{1 / 2}}-\frac{1}{(x+2)^{5 / 6} x^{1 / 2}}+\frac{1}{2^{1 / 2} x^{5 / 6}}\right\} \mathrm{d} x,
\end{gathered}
$$

and $I_{1}$ is plotted against $z$ in Figure 4 . When $z$ is small compared to unity, $I_{1}$ can be neglected.

Next, we can integrate Equation (26) to obtain

$$
\begin{gathered}
u^{(0)}=\frac{\left.\mathrm{os}^{(0)}, x, t\right)}{\partial s_{i j}} s_{i j}^{(1)}+ \\
+6 \int_{y_{\mathrm{b}}}^{y}\left(\sigma_{x y}^{(0)^{3}}+s_{x x}^{(0)^{2}} \sigma_{x y}^{(0)}\right) \mathrm{d} y
\end{gathered}
$$

although we do not yet know $s_{\not x}^{(1)}$. (Note that the standard summation convention has been adopted in the first term of Equation (37).) We can find this last quantity, if needed, by differentiating Equation (37) and inserting the result into Equation (25). Again, this may give a differential equation or an algebraic equation depending on whether $\Phi$ depends on $s_{x x}$ or not.

Finally, we can use incompressibility to find

$$
v^{(0)}=\left(y_{\mathrm{b}}-y\right) \mathrm{d} u^{(-1)} / \mathrm{d} x,
$$

$$
v^{(1)}=y_{b}(x) u^{(-1)}(x)-\int_{y_{\mathrm{b}}}^{y}\left(\partial u^{(0)} / \partial x\right) \mathrm{d} y .
$$

It is of some interest to consider the solution when $\Phi\left(s^{(0)}, x, t\right)$ becomes small compared to unity. In that case, $r$ in Equation (35) will be small and we choose to retain only the largest terms. We find

$$
s_{x x}^{(0)}=\frac{1}{3 \sigma_{x y}^{(0)^{2}}} \frac{\mathrm{d} \Phi}{\mathrm{d} x}\left(\mathrm{~s}^{(0)}, x, t\right)
$$

just as it would be in the preyious case when $v \ll \delta$, except now the smallness of $s_{x x}(0)$ is determined by the magnitude of $\Phi$. The significant term in Equation (37) is now

$$
6 \int_{y_{\mathrm{b}}}^{y} \sigma_{x y}^{(0)^{3}} \mathrm{~d} y .
$$

This integrates to give $\frac{3}{2}\left[h^{4}-\left(y_{\mathrm{s}}-y\right)^{4}\right] \sin ^{3} \alpha$ and so the solution is essentially the same as Equations (20). Thus we see that the results for the case where the leading term in the velocity $u$ is $O(\Phi / \delta)$ behave satisfactorily when $\Phi$ becomes small and the standard and intermediate solution can be recovered. In fact, the solution of Equations (30) and (33) through (39) contains the solution for magnitudes of the basal velocity ranging from $O(1 / \delta)$ down to $O(1)$ and less, and thus one solution can be used for this entire range.

It is important to note that in the solutions obtained so far the down-slope component of weight is supported by the basal shear stress to leading order. In general, the higher-order terms will alter the basal shear stress so that it is not exactly equal to the down-slope weight, but of course this correction is small. As mentioned previously, the expansion breaks down in certain circumstances. Consider sliding laws of the kinds shown in Figure 1. Assume that for some reason the basal shear stress, computed to leading order as $h \sin \alpha$, is comparable with $\tau_{\mathrm{m}}$. This could occur because of high lubrication due to excess water in the melt season, which could cause the glacier to slide relatively rapidly at low basal shear stress. In regions moving slower than $u_{b}^{1}, h \sin \alpha<T_{m}$ and, as long as that condition is met, the preceding solutions can be used. However, as $h$ sin $\alpha$ approaches $\boldsymbol{T}_{m}$, say in a thicker portion of the glacier, then $\partial \Phi / \partial \tau_{b}$ becomes very large. In fact, at $1, \partial \phi\left\langle\partial \tau_{b}=\right.$ $\infty$ and therefore according to Equation (37) $u^{(0)} \rightarrow \infty$. This means that $u(0)$ will exceed the leading-order approximation for velocity $u(-1) / \delta=\Phi / 6$ and the expansion has broken down. In addition, if $h \sin \alpha$ exceeds $\tau_{\mathrm{m}}$ as it would do in general for sliding speeds exceeding $u_{b}^{1}$ between points 1 and 2 in Figure 1 , then the down-slope component of weight can no longer be supported by basal shear stress to leading order. Since the leading-order equations can no longer be satisfied, the perturbation expansion has broken down and the approximations are no longer valid. Of course, if the sliding speed exceeds $u_{\mathrm{b}}^{2}$ in Figure 1 , then with sliding law (a) the basal shear stress could match $h \sin \alpha$ once more. Because the velocity is a continuous function of position, there may be a substantial portion of the glacier sliding at speeds between 1 and 2 and, if we are dealing with law (b), there could be large portions of the glacier where $h$ sin $\alpha$ exceeds $\tau_{\mathrm{b}}$. Thus it is interesting to consider such a possibility and this is dealt with in the next section.

\section{RAPID STRETCHING: LONGITUDINAL STRESS} GRADIENT SUPPORTS PART OF THE WEIGHT

Beyond the peak in Figure 1, some portion of the down-slope component of weight is borne by the longitudinal stress gradient to leading order. As this is not the case in the results discussed so far, we must seek another solution. We shall study now the situation where $v \ll \delta$ so that $s_{x x}$ dominates $\sigma_{x y}$. In particular, we are considering the case in which some of the glacier's weight is supported by $\sigma_{x x}$ and we see from the equilibrium Equations (1) that this requires

$$
\begin{aligned}
& \sigma_{x x}=(1 / \delta) \sigma_{x x}^{(-1)}+\sigma_{x x}^{(0)}+O(\delta), \\
& \sigma_{x y}=\sigma_{x y}^{(0)}+\delta \sigma_{x y}^{(1)}+O\left(\delta^{2}\right), \\
& \sigma_{y y}=\sigma_{y y}^{(0)}+\delta \sigma_{x y}^{(1)}+O\left(\delta^{2}\right),
\end{aligned}
$$

from which it follows that

$$
s_{x x}=(1 / \delta) \frac{1}{2} \sigma_{x x}^{(-1)}+O(1) \text {. }
$$

As before $\sigma_{y y}^{(0)}=\left(y-y_{\mathrm{b}}\right) \cos \alpha$. From the flow law, Equation (3), we find that

$$
\delta \partial u / \partial x=\left[\frac{3}{8 \delta^{3}}\right]\left(\sigma_{x x}^{(-1)}\right)^{3}+O\left(18^{3}\right)
$$




$$
\partial u / \partial y=\left[\frac{3}{2 \delta^{2}}\right]\left(\sigma_{x x}^{(-1)}\right)^{2} \sigma_{x y}^{(0)}+O\left(1 \delta^{2}\right) .
$$

Consequently, these can be satisfied to leading order if we require

$$
u=\left(1 / \delta^{4}\right) u^{(-4)}(x)+O\left(1 / \delta^{3}\right)
$$

giving $\mathrm{d} u^{(-4)} / \mathrm{d} x=3\left(\sigma_{x x}^{(-1) 3}\right) / 8$. Since $u^{(-4)}$ is independent of $y$, this shows that $\sigma_{x x}(-1)$ is also independent of depth. It should be noted that the velocity has increased to $O(1 / \delta)^{4}$ and this must happen for regions beyond the peak in Figure 1. Furthermore, from Equation (43) we now see that the case in which $s_{x x}$ supports part of the weight is consistent with a sliding law for which $v=O\left(\delta^{4}\right)$. Therefore, for this solution we will identify $v$ with $\delta^{4}$, without loss of generality.

From the equilibrium Equation (1a) it follows that

$$
\frac{\partial \sigma_{x y}^{(0)}}{\partial y}+\frac{\mathrm{d} \sigma_{x x}^{(-1)}}{\mathrm{d} x}=-\sin \alpha
$$

and thus

$$
\sigma_{x y}^{(0)}=\sigma_{x y}^{(0)}\left(x, y_{\mathrm{b}}\right)-\left(y-y_{\mathrm{b}}\right)\left[\sin \alpha+\frac{\mathrm{d} \sigma_{x x}^{-1}}{\mathrm{~d} x}\right] .
$$

The boundary condition at $y=y_{\mathrm{S}}$ is given by

$$
\sigma_{x y}^{(0)}\left(x, y_{\mathrm{s}}\right)=y_{\mathrm{s}}{ }^{\prime}(x) \sigma_{x x}^{(-1)}(x)
$$

and together with Equation (45) this gives

$$
\begin{aligned}
& \frac{\mathrm{d}}{\mathrm{d} x}\left(h \sigma_{x x}^{(-1)}\right)+h \sin \alpha-\sigma_{x y}^{(0)}\left(x, y_{\mathrm{b}}\right)+ \\
& +y_{\mathrm{b}}^{\prime}(x) \sigma_{x x}^{(-1)}=0 .
\end{aligned}
$$

If we restate the sliding law as

$$
u^{(-4)}(x)=\Phi\left(\sigma_{x y}^{(0)}\left(x, y_{\mathrm{b}}\right)\right)
$$

where $\Phi$ is $O(1)$ we can consider the inversion

$$
\sigma_{x y}^{(0)}\left(x, y_{\mathrm{b}}\right)=\Phi^{-1}\left(u^{(-4)}(x)\right)
$$

in which we take $\Phi^{-1}$ to be a single-valued function of $u^{(-4)}$ as it would be in the two cases illustrated in Figure 1.

The differential Equation (46) must be solved with appropriate boundary conditions. If we consider a segment $A B$ as in Figure 3 in which this solution is valid, then boundary conditions are required at $\mathrm{A}$ and B. Outside $A B$, we shall assume that the ice is moving slowly and the down-slope component of weight is supported by the basal shear stress to leading order.

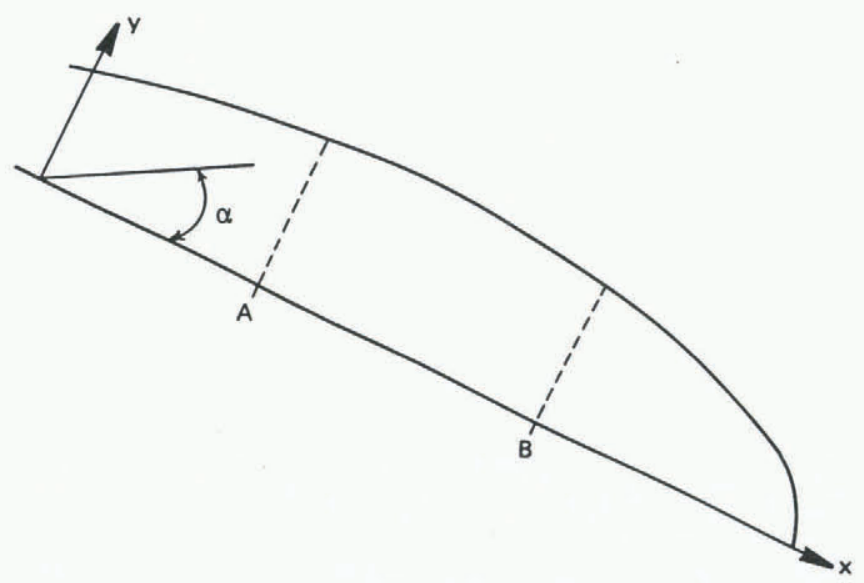

Fig. 3. Schematic of a rapidly sliding glacier segment constrained by slowly moving portions.
This means that the basal velocity is $O(1 / \delta)$ at most outside AB. Near $A$ and $B$ the perturbation approximation for basal velocity of $O(1 / \delta)$ will break down. However, we shall assume that the ice velocities are $O(1 / 8)$ at $A$ and $B$. This means that $u^{(-4)}$ in AB must vanish at $\mathrm{A}$ and $\mathrm{B}$, giving us an ad hoc matching to the more slowly moving ice on either side. This somewhat ad hoc matching condition is consistent with mass conservation and assumes that the local regions near A and $B$ are relatively passive. Rigorously derived matching conditions would require the determination of the local solutions valid near the transition points $A$ and $B$ and this is not an easy task in the present problem. However, in defense of the approach taken here, it is quite common in perturbation problems of this type to find that the local solutions are passive. Therefore assuming $u^{(4)}$ vanishes at $\mathrm{A}$ we then note that

$$
u^{(-4)}(x)=\int_{x_{A}}^{x} \frac{3}{8}\left(\sigma_{x x}^{(-1)}\right)^{3} \mathrm{~d} x,
$$

since $\mathrm{d} u^{(-4)} / \mathrm{d} x=3\left(\sigma^{(-1)}\right)^{3} / 8$. Consequently, since $\sigma_{x y}^{(0)}$ is related to $u^{(-4)}(x)$ by Equation (48) and therefore to $\sigma_{x}(-1)$ by Equation (49), we see that Equation (46) is an integro-differential equation for $\sigma_{x x}(-1)$. To solve it we would have to apply the boundary condition $u^{(-4)}\left(x_{\mathrm{B}}\right)=0$.

The integro-differential Equation (46) involves the balance between the body force $h \sin \alpha$, the stress resultant $h \sigma_{x x}(-1)$, the basal shear stress, and an effective basal shear stress $y_{\mathrm{b}}^{\prime}(x)\left(\rho^{(-1)}\right)$ due to the slope of the base. The value of $h \sigma_{x x}$ at $A$ and $B$ will give the forces exerted by the rapid sliding region on the slowly moving ice on either side. The longitudinal stress involved in this thrust will be much larger than those involved in the solution for slow motion of the glacier. The forces involved will be transmitted to the base of the slowly moving sections and supported by shear stress over a physical length comparable to thickness $\hat{h}$ on either side of $A B$. Thus the sections on either side of $A$ and $B$ act as shear-stress concentrators. These shear stresses will tend to cause the total basal values to exceed the critical value $\tau_{m}$ and thus cause the rapid sliding region to spread. In fact, the process would be similar to the spreading of shear bands in clay which obey a shear-stress-shear-velocity jump law across the band like curve (b) in Figure 1 as discussed by Palmer and Rice (1973). This is used as the basis for a surge model in our related paper.

\section{GLACIERS ON A GENTLE SLOPE}

In the previous sections we have been concerned with glaciers moving down moderate slopes. We will now consider the case of gentle slopes. When the average slope for the glacier is $O(\delta)$, we can rewrite $\sin \alpha$ as $\beta \delta$ and $\cos \alpha$ as $\sqrt{1-\delta^{2} \beta^{2}}$ where $\beta=O(1)$. The standard solution corresponding to large shear stress compared to longitudinal deviatoric stress then becomes

$$
\left.\begin{array}{rl}
\sigma_{x x} & =y-y_{\mathrm{s}}+O\left(\delta^{2}\right), \sigma_{y y}=y-\mathrm{y}_{\mathrm{s}}+O\left(\delta^{2}\right) \\
\sigma_{x y} & =\delta y\left(y-y_{\mathrm{s}}\right)+O\left(\delta^{2}\right) \\
\text { where } y=-\beta+y_{\mathrm{s}}^{\prime}(x), \\
u=\Phi\left(\tau_{\mathrm{b}}, x, t\right)+\frac{3}{2} \delta^{3} y^{3}\left[\left(y_{\mathrm{s}}-y\right)^{4}-h^{4}\right]+O\left(\delta^{6}\right)
\end{array}\right\}
$$

where for consistency $\Phi=O\left(\delta^{3}\right)$. As before, there is a boundary layer near the free surface as discussed by Johnson and McMeeking (1984). Note that, since the slope is small, the force due to gravity which drives the ice down the incline is of the same order of magnitude as the driving force due to gravity which causes the ice to flow from deep to shallow regions. Furthermore, in the standard solution both of these are balanced by the shear stress and the longitudinal deviatoric stress is higher order. 
If, on the other hand, we consider the case when $s_{x x}=O(\delta)$ and therefore comparable to $\sigma_{x y}$, we then

$\sigma_{y y}=\sigma_{y y}^{(0)}+O\left(\delta^{2}\right), \quad \sigma_{x y}=\delta \sigma_{x y}^{(1)}+\delta^{2} \sigma_{x y}^{(2)}+O\left(\delta^{3}\right)$,

$s_{x x}=\delta s_{x x}^{(1)}+O\left(\delta^{2}\right), \quad u=\delta^{2} u^{(2)}+\delta^{3} u^{(3)}+O\left(\delta^{4}\right)$

where $\sigma_{y y}^{(0)}=\left(y-y_{\mathrm{s}}\right), \sigma_{x y}^{(1)}=-y\left(y_{s}-y\right)$, and from $u$ we see that this solution corresponds to one in which $\Phi$ $=O\left(\delta^{2}\right)$. In addition, from the flow law we find that the leading prder term in $u$ is such that $u^{(2)}=u^{(2)}(x)$ and then $s_{x x}^{(1)}$ is the solution of the cubic

$$
3\left(s_{x x}^{(1)^{2}}+\sigma_{x y}^{(1)^{2}}\right) s_{x x}^{(1)}-\mathrm{d} u^{(2)} / \mathrm{d} x=0 .
$$

This is very similar to Equation (34) and when $\delta^{2} u^{(2)}(x)$ $=\Phi\left(6 \tau_{b}(1)\right)$ depends only on the basal shear stress. The cubic equation will have a solution of the form of Equation (35). At higher order we find

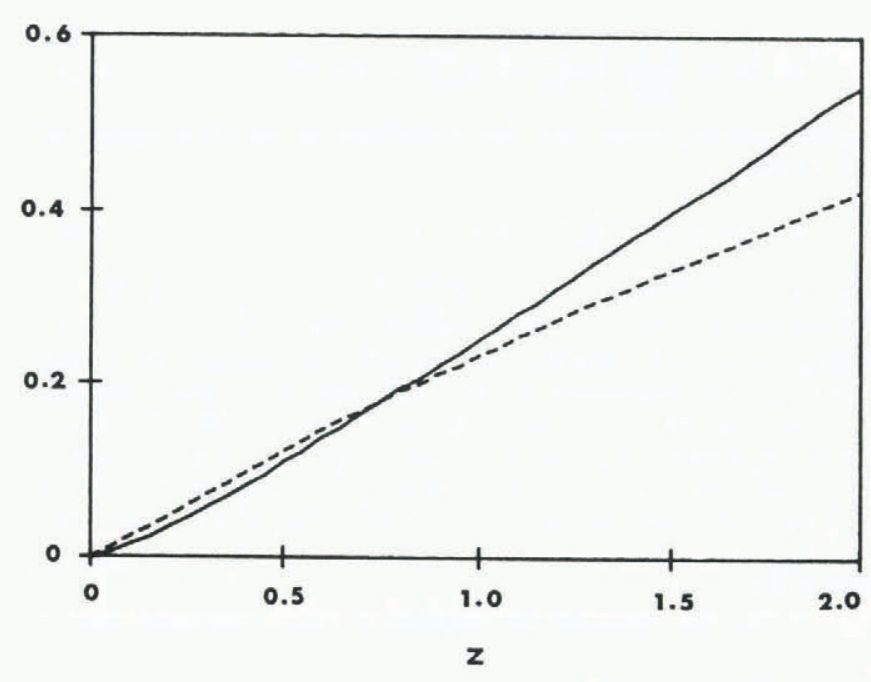

Fig. 4. Functions arising in expressions for stress in glaciers. The solid line is $I_{1}(z)$ and the dashed line is $I_{2}(z)$.

$$
\begin{gathered}
\sigma_{x y}^{(2)}=y_{S}^{\prime}(x)\left[4 s_{x x}^{(1)}\left(x, y_{\mathrm{S}}\right)-2 s_{x x}^{(1)}(x, y)\right]- \\
-\frac{2 y_{\mathrm{S}}^{\prime \prime}(x)}{y}\left[\left(y-y_{\mathrm{S}}\right) s_{x x}^{(1)}(x, y)+\frac{1}{3} \frac{s_{x x}^{(1)}\left(x, y_{\mathrm{S}}\right)}{2^{1 / 3} \bar{a} 1 / 6}\left(2^{1 / 6} z^{1 / 2}-\frac{6}{\sqrt{2}} z^{1 / 6}+I_{2}(z)\right)\right]- \\
-\left.\frac{s_{x x}^{(1)}\left(x, y_{\mathrm{S}}\right)}{\sqrt{3} 2^{2 / 3} y} \frac{\partial s_{x x}^{(1)} \mid}{\partial x}\right|_{y=y_{\mathrm{S}}}\left[2^{1 / 6} z^{1 / 2}+\frac{6}{\sqrt{2}} z^{1 / 6}+I_{1}(z)\right] \\
I_{2}(z)=\int_{0}^{z}\left\{\frac{x+1}{(x+2)^{1 / 2} x^{5 / 6}}-\frac{1}{2^{1 / 2} x^{5 / 6}}-\frac{x+1}{(x+2)^{5 / 6} x^{1 / 2}}+\frac{1}{2^{5 / 6} x^{1 / 2}}\right\} \mathrm{d} x,
\end{gathered}
$$

where $z=\left[\bar{a}\left(y_{\mathrm{S}}-y\right)^{6}+1\right]^{1 / 2}-1$,

$$
\bar{a}=\frac{4}{27}\left\{\frac{y}{s_{x x}^{(1)}\left(x, y_{\mathrm{s}}\right)}\right\}^{6},
$$

and $I_{1}(z)$ is given in Equations (36). $I_{1}$ and $I_{2}$ are plotted against $z$ in Figure 4 . When $z$ is small compared to unity, $I_{1}$ and $I_{2}$ can be neglected. In addition

$$
\begin{aligned}
u^{(3)} & =\frac{1}{\delta^{2}} \Phi^{\prime}\left(\delta \tau_{\mathrm{b}}^{(1)}\right)\left(\delta \tau_{\mathrm{b}}^{(1)}\right) \tau_{\mathrm{b}}{ }^{(2)}+3 s_{x x}^{(1)^{2}} y\left[\left(y-y_{\mathrm{s}}\right)^{2}-h^{2}\right]+ \\
& +\frac{3}{2} y^{3}\left[\left(y-y_{\mathrm{s}}\right)^{4}-h^{4}\right] .
\end{aligned}
$$

Note that this solution contains the standard solution as a special case since it can be recovered by allowing $\Phi$ to become smaller than $O\left(\delta^{2}\right)$.

We can also consider the situation when $\Phi=O(\delta)$ which corresponds to large longitudinal deviatoric stresses compared to shear stress. However, again the shear stress will be balancing the driving forces due to gravity. In this case we have with that of $u^{(1)^{\prime}}(x)$. We assume that $u^{(1)}$ is determined by the basal shear stress and that $s_{x x}^{(1)}$ can be determined in turn from this. At higher order we find

$\sigma_{x y}^{(2)}=2 s_{x x}^{(1)}{ }^{\prime}(x)\left(y_{s}-y\right)+2 s_{x x}^{(1)}(x) y_{S}^{\prime}(x)$.

Note that $u^{(2)}=u^{(2)}(x)$ and shearing flow appears only at $O\left(\delta^{7 / 3}\right)$. A similar solution can be found for $\Phi=O(1)$ in which case $u=O(1)$ and $s_{x x}=O\left(\delta^{1 / 3}\right)$. However, we will consider next the situation when $\Phi$ is large and the forces due to gravity are not balanced by the shear stress alone.

In this situation

$$
\begin{aligned}
& \sigma_{y y}=\sigma_{y y}^{(0)}+O\left(\delta^{2}\right), \quad \sigma_{x y}=\delta \sigma_{x y}^{(1)}+O\left(\delta^{2}\right), \\
& s_{x x}=s_{x x}^{(0)}+O(\delta), \quad u=\frac{1}{\delta} u^{(-1)}+O(1), \\
& \text { and } \Phi=O(1 / \delta),
\end{aligned}
$$

with $\sigma_{y y}^{(0)}=y-y_{\mathrm{S}}$ and $u^{(-1)}=u^{(-1)}(x)$. Also

$$
\left.\begin{array}{c}
\sigma_{y y}=\sigma_{y y}^{(0)}+O\left(\delta^{2}\right), \quad \sigma_{x y}=\delta \sigma_{x y}^{(1)}+\delta^{5 / 3} \sigma_{x y}^{(2)}+O\left(\delta^{7 / 3}\right), \\
s_{x x}=\delta^{2 / 3} s_{x x}^{(1)}+\delta^{4 / 3} s_{x x}^{(2)}+O\left(\delta^{2}\right), \quad u=\delta u^{(1)}+\delta^{5 / 3} u^{(2)}+O\left(\delta^{7 / 3}\right),
\end{array}\right\}
$$

with $\sigma_{y y}^{(0)}=y-y_{\mathrm{S}}, \sigma_{x y}^{(1)}=-\gamma\left(y_{\mathrm{S}}-y\right), u^{(1)}=u^{(1)}(x)$

and $s_{y y}^{(1)}= \pm\left|\frac{u^{(1) '}(x)}{3}\right|^{1 / 3}$ with the sign chosen to agree

$$
\begin{aligned}
s_{x x}^{(0)} & =s_{x x}^{(0)}(x)= \pm\left|\frac{u^{(-1)^{\prime}(x)}}{3}\right|^{1 / 3} \text { and } \\
\sigma_{x y}^{(1)} & \left.=\left(-\beta+y_{s}^{\prime}(x)\right)\left(y-y_{\mathrm{s}}\right)-2 s_{x x}^{(0)}\right)^{\prime}(x)\left(y-y_{\mathrm{s}}\right)+ \\
& +2 s_{x x}^{(0)}(x) y_{\mathrm{S}}^{\prime}(x)
\end{aligned}
$$


which gives at the base

$$
\begin{aligned}
& \sigma_{x y}^{(1)}\left(x, y_{\mathrm{b}}\right)=-h\left(-\beta+y_{\mathrm{s}}^{\prime}(x)\right)+ \\
+ & \left.2 h s_{x x}^{(0)}\right)^{\prime}(x)+2 s_{x x}^{(0)}(x) y_{\mathrm{s}}^{\prime}(x) .
\end{aligned}
$$

If the velocity at the bed depends on the shear stress, Equation (59) is an integro-differential equation in $x$ and it must be solved with boundary conditions on the similar to the steep-slope case.

\section{CORRECTED SHEAR STRESS AT THE BASE}

We will now consider how the longitudinal stress gradient influences the shear stress at the base by introducing corrections to the leading-order estimate Attempts have been made by various workers (Robin, 1967; Collins, 1968; Nye, 1969; Budd, 1970; Hutter, 1981) to find this relationship in connection with experiments to relate bottom and surface topography. Their technique is to start with the exact equations, integrate through the glacier thickness and make assumptions about dropping terms and then invoke the constitutive law. The simplest form of their result can be stated as

$$
\hat{\tau}_{\mathrm{b}}=\hat{\sigma}_{x y}\left(\hat{x}, \hat{y}_{\mathrm{b}}\right)=\rho g \hat{h} \sin \alpha+G-T
$$

where $T$ is the shear stress $\sigma_{x y}$ integrated twice through the thickness and

$$
G= \pm 2 \hat{h} \frac{\mathrm{d}}{\mathrm{d} \hat{x}}\left|\frac{\partial \hat{u}\left(\hat{x}, \hat{y}_{\mathrm{S}}\right)}{3 B \partial \hat{x}}\right|^{1 / 3}
$$

with the sign chosen to agree with that of $\partial \hat{u}\left(\hat{x}, \hat{y}_{\mathrm{S}}\right) / \partial \hat{x}$. By comparing our results for various flow regimes to Equation (60) wse can determine when their assumptions are appropriate and provide better results for situations when their assumptions fail.

First, we consider the standard case for the steep glacier which would be valid when it is stuck to the bedrock. This is the case considered by Nye (1969). From the first of Equations (9) we find stress or velocity given at two values of $x$ in a manner

stress deviator. Twice the longitudinal gradient of this stress integrated through the thickness of the boundary layer must be balanced by a shear stress at the bottom of the layer. To the underlying ice, this stress appears as if it is a non-zero shear stress at the upper free surface. Johnson and McMeeking (1984) have shown that the boundary-layer thickness is $\delta^{1 / 3} \hat{h}$ so with $\hat{y}_{s}^{\prime}(\hat{x})=0$

$$
\hat{\tau}_{\mathrm{b}}=\rho g \hat{h} \sin \alpha+2 \int_{\left(\hat{y}_{\mathrm{S}}-\delta^{1 / 3} \hat{h}\right)}^{\hat{y}_{\mathrm{S}}} \frac{\partial \hat{s}_{x x}}{\partial \hat{x}} \mathrm{~d} \hat{x}
$$

which is almost identical to the results of Collins and Nye except that the integration is carried out only in the boundary layer. The last term in Equation (61) is an evaluation of this integral.

In the case of the standard glacier solution on the gentle slope, Johnson and McMeeking (1984) did not carry out the matching to sufficient terms for the result we wish. However, the situation is likely to be similar to the steepslope case.

Next consider the case when there is substantial sliding at the bed. For the steep glacier which is stretching rapidly so that the longitudinal deviatoric stress is comparable to the shear stress, we find from Equations (33) and (36) that when $\alpha$ is chosen so that $y_{\mathrm{s}}^{\prime}(x)=0$

$\hat{\tau}_{\mathrm{b}}=\rho g \hat{h} \sin \alpha+\frac{\left\{\frac{1}{3 B} \frac{\partial \hat{u}\left(\hat{x}, \hat{y}_{\mathrm{S}}\right)}{\partial \hat{x}}\right\}^{1 / 3} \frac{\partial}{\partial \hat{x}}\left|\frac{1}{3 B} \frac{\partial \hat{u}\left(\hat{x}, \hat{y}_{\mathrm{S}}\right)}{\partial \hat{x}}\right|^{1 / 3}}{\sqrt{3} 2^{2 / 3} \rho g \sin \alpha}$

$$
\left[2^{1 / 6} z^{1 / 2}+6 z^{1 / 6} / \sqrt{2}+I_{1}\right](64)
$$

where

$$
z=\sqrt{\frac{4}{27}(\rho g \hat{h} \sin \alpha)^{6} /\left\{\frac{1}{3 B} \frac{\left.\partial \hat{u}(\hat{x}, \hat{y})_{\mathrm{S}}\right)}{\partial \hat{x}}\right\}^{2}+1}-1
$$

$$
\hat{\tau}_{\mathrm{b}}=\hat{\sigma}_{x y}\left(\hat{x}, \hat{y}_{\mathrm{b}}\right)=\rho g \hat{h}\left(\sin \alpha-\hat{y}_{\mathrm{s}}^{\prime}(\hat{x}) \cos \alpha\right) \pm \frac{3 k}{2 \rho g \sin \alpha} \frac{\partial}{\partial \hat{x}}\left|\frac{\partial \hat{u}_{\mathrm{S}}\left(\hat{x}, \hat{y}_{\mathrm{S}}\right)}{B \partial \hat{x}}\right|^{2 / 3}
$$

with the sign chosen to agree with that of $\partial \hat{u}_{\mathrm{s}}\left(\hat{x}, \hat{y}_{\mathrm{s}}\right) / \partial \hat{x}$. Equation (61) can be simplified by choosing $\alpha$ so that $y_{s}^{\prime}(\hat{x})$ disappears at the point of interest, as did Nye. We see that the term $T$ is not present since it is $O\left(\delta^{2}\right)$ and we have kept only terms $O\left(8^{5 / 3}\right)$. In any case, Johnson and McMeeking (1984) have not carried out the matching necessary to evaluate terms of $O\left(\delta^{2}\right)$. Following Collins (1968) and Budd (1970), Nye dispensed with $T$ on empirical grounds. We see, however, that the assumption of Collins and Nye which leads to the term $G$ in Equation (60) is invalid for this case. The gradient term in Equation (61) is $G$ multiplied by

$$
\frac{\left.3^{5 / 3} k \mid\left(\partial \hat{x}_{\mathrm{s}} \hat{x}, \hat{y}_{\mathrm{s}}\right) / \partial \hat{x}\right) /\left.3 B\right|^{1 / 3}}{2 \rho g \hat{h} \sin \alpha}
$$

or $3^{5 / 3} k / 2$ times the ratio of the surface longitudinal stress to the basal shear stress estimated to leading order. The parameter $k$ is given in Equation (9a). Of course, Budd, Collins, and Nye were unaware of the boundary layer and instead tried to estimate the relationship between the average longitudinal deviatoric stress and the surface strainrate to obtain their $G$.

The influence of the boundary layer on shear stress can be understood from the following simple model. Consider the region near the free surface. It is stretching or compressing to leading order and this requires a longitudinal and $I_{1}$ is plotted versus $z$ in Figure 4 . It can be seen that the result for this case bears some similarity to the standard glacier formula of Equation (61). In addition, from Equation (53) we see that the result for a glacier on a gentle slope in which the longitudinal deviatoric stress is comparable with the shear stress (with $\alpha$ chosen to make $\hat{y}_{s}^{\prime}(\hat{x})=0$ ) is the same as Equation (64) with the additional term

$$
\begin{aligned}
2 \hat{y}_{\mathrm{S}}^{\prime \prime}(\hat{x}) & {\left[-\frac{1}{2 \sqrt{3}} \frac{\left\{\frac{1}{3 B} \frac{\partial \hat{u}\left(\hat{x}, \hat{y}_{\mathrm{S}}\right)}{\partial \hat{x}}\right\}^{2 / 3}}{\rho g \sin ^{2} \alpha}\left[2^{1 / 6} z^{1 / 2}-\frac{6}{\sqrt{2}} z^{1 / 6}+I_{2}(z)\right]+\right.} \\
& \left.+\frac{\left(\hat{y}-\hat{y}_{\mathrm{S}}\right) \hat{s}(1)\left(\hat{x}, \hat{y}_{\mathrm{b}}\right)}{\sin \alpha}\right]
\end{aligned}
$$

where $s_{x x}^{(1)}\left(\hat{x}, \hat{y}_{\mathrm{b}}\right)$ must be obtained from the solution to Equation (52) at the base.

Another solution on the gentle slope is given by Equation (55), where the leading-order velocity is $O(8)$ and is independent of depth, but the down-slope component of weight is supported by basal shear stress to leading order. In this case, the longitudinal deviatoric stress is $O\left(1 / 8^{1 / 3}\right)$ larger than the shear stress. From Equations (55) and (56) we find that, when $\alpha$ is chosen so that $\hat{y}_{s}^{\prime}(\hat{x})=0$, the shear 
stress at the base is given by Equation (60) with $T$ omitted. Thus the estimate of Nye, Collins, and Budd is valid (omitting $T$ ) when the velocity at the base of a glacier on a gentle slope is $O(\delta)$ and therefore the glacier is stretching rapidly, but cannot be true when the base of the glacier is stuck to the bedrock, contradicting a suggestion of Nye.

Finally, we consider the case where the down-slope component of weight is partly borne by the longitudinal stress gradient to leading order. The fact that the leadingorder longitudinal deviatoric stress is independent of depth makes it clear that Equation (60) with $T$ omitted gives the corrected basal shear stress both for the gentle and steep cases.

\section{SUMMARY}

In the present paper we have examined glacial flow in cases where the longitudinal deviatoric stress becomes large. In particular, we have developed the theory for the case when the longitudinal deviatoric stress is comparable to the shear stress and the case when the longitudinal stress is the dominant stress component. In each case the stress state is strongly influenced by the character of the basal sliding. Furthermore, the stress state in these cases differs fundamentally from that of the standard solution where the shear stress is dominant and supports the weight. Lastly, we examined the effect of the longitudinal stress on the basal shear stress and compared the results with previous work on relating surface and bottom topography.

\section{ACKNOWLEDGEMENT}

This research was carried out while the authors were supported by National Science Foundation grants MEA 82-11018 (RMM) and MEA 83-11495 (REJ).

\section{REFERENCES}

Budd, W.F. 1970. The longitudinal stress and strain-rate gradients in ice masses. Journal of Glaciology, Vol. 9, No. 55 , p. $19-27$.

Budd, W.F. 1975. A first simple model for periodically self-surging glaciers. Journal of Glaciology, Vol. 14, No. 70 , p. $3-21$.
Colbeck, S.C., ed. 1980. Dynamics of snow and ice masses. New York, etc., Academic Press.

Collins, I.F. 1968. On the use of the equilibrium equations and flow law in relating the surface and bed topography of glaciers and ice sheets. Journal of Glaciology, Vol. 7, No. 50 , p. $199-204$.

Fowler, A.C. 1982. Waves on glaciers. Journal of Fluid Mechanics, Vol. 120, p. 283-321.

Hutter, K. 1981. The effect of longitudinal strain on the shear stress of an ice sheet: in defence of using stretched coordinates. Journal of Glaciology, Vol. 27, No. 95, p. 39-56.

Hutter, K. 1982[a]. Dynamics of glaciers and large ice masses. Annual Review of Fluid Mechanics, Vol. 14, p. 87-130.

Hutter, K. 1982[b]. Glacier flow. American Scientist, Vol. 70 , p. $26-34$.

Hutter, K. [ $\left.{ }^{\mathrm{c}} 1983.\right]$ Theoretical glaciology; material science of ice and the mechanics of glaciers and ice sheets. Dordrecht, etc., D. Reidel Publishing Company/Tokyo, Terra Scientific Publishing Company.

Johnson, R.E., and McMeeking, R.M. 1984. Near-surface flow in glaciers obeying Glen's law. Quarterly Journal of Mechanics and Applied Mathematics, Vol. 37, Pt. 2, p. 273-91.

Lliboutry, L.A. 1968. General theory of subglacial cavitation and sliding of temperate glaciers. Journal of Glaciology, Vol. 7, No. 49, p. 21-58.

Morland, L.W., and Johnson, I.R. 1980. Steady motion of ice sheets. Journal of Glaciology, Vol. 25, No. 92, p. 229-46.

Morland, L.W., and Johnson, I.R. 1982. Effects of bed inclination and topography on steady isothermal ice sheets. Journal of Glaciology, Vol. 28, No. 98, p. 71-90.

Nye, J.F. 1969. The effect of longitudinal stress on the shear stress at the base of an ice sheet. Journal of Glaciology, Vol. 8, No. 53, p. 207-13.

Palmer, A.C., and Rice, J.R. 1973. The growth of slip surfaces in the progressive failure of over-consolidated clay. Proceedings of the Royal Society of London, Ser. A, Vol. 332, No. 1591, p. 527-48.

Raymond, C.F. 1980. Temperate valley glaciers. (In Colbeck, S.C., ed. Dynamics of snow and ice masses. New York, etc., Academic Press, p. 79-139.)

Robin, G. de Q. 1967. Surface topography of ice sheets. Nature, Vol. 215, No. 5105, p. 1029-32. 